\title{
Large Deviations of Probability Rank
}

\author{
Erdal Arikan \\ Electrical Eng. Dept. \\ Bilkent University \\ 06533 Ankara, Turkey \\ arikancee.bilkent.edu.tr
}

\begin{abstract}
Consider a pair of random variables $(X, Y)$ with distribution $P$. The probability rank function is defined so that $G(x \mid y)=1$ for the most probable outcome $x$ conditional on $Y=y, G(x \mid y)=2$ for the second most probable outcome, and so on, resolving ties between elements with equal probabilities arbitrarily. The function $G$ was considered in [1] in the context of finding the unknown outcome of a random experiment by asking questions of the form 'Is the outcome equal to $x$ ?' sequentially until the actual outcome is determined. The primary focus in [1], and the subsequent works [2], [3], was to find tight bounds on the moments $\mathrm{E}\left[G(X \mid Y)^{\theta}\right]$. The present work is closely related to these works but focuses more directly on the large deviations properties of the probability rank
\end{abstract} function.

\section{RESULTS}

The aim of this work is to determine the large deviation exponent of $\ln G$,

$$
\lim _{n \rightarrow \infty} n^{-1} \ln P\left[\ln G\left(X^{n} \mid Y^{n}\right)>n L\right],
$$

for a sequence of pairs of r.v.'s $\left(X^{n}, Y^{n}\right)$ under various assumptions regarding their distribution. Special instances of this problem correspond to finding the error exponent in source and channel coding problems of information theory. E.g., if we regard $X^{n}$ as an input of length $n$ to a noisy channel and $Y^{n}$ as the channel output, $P\left[\ln G\left(X^{n} \mid Y^{n}\right)>n L\right]$ is the probability of decoding error for a list decoder with list size $e^{n L}$. We begin by noting that the mean of $\ln G$ is closely related to the Shannon entropy.

Proposition 1 For $(X, Y)$ a pair of jointly distributed random variables,

$$
-\ln (1+\ln M)+H(X \mid Y) \leq \mathrm{E}[\ln G(X \mid Y)] \leq H(X \mid Y)
$$

where $M$ is the maximum over all $y$ of the range of $X$ conditioned on $Y=y$.

We study large-deviations of $\ln G\left(X^{n} \mid Y^{n}\right)$ under the assumption that the sequence of functions

$$
\varphi_{n}(\theta) \triangleq \frac{1}{n} \ln \mathbf{E}\left[G\left(X^{n} \mid Y^{n}\right)^{\theta}\right]
$$

converges to a limit $\varphi(\theta)$. We let $R_{\varphi^{\prime}}$ denote the range of $\varphi^{\prime}$. Now, the Gärtner-Ellis theorem $[4, \mathrm{p} .15]$ gives

Proposition 2 For any $L \in R_{\varphi^{\prime}}$

$$
\begin{aligned}
& \lim _{n \rightarrow \infty} n_{-1} \ln P\left[\ln G\left(X^{n} \mid Y^{n}\right)>n L\right]=\varphi\left(\theta_{L}\right)-\theta_{L} \varphi^{\prime}\left(\theta_{L}\right) \\
& \text { where } \theta_{L}=\inf \left\{\theta: \varphi^{\prime}(\theta)=L\right\} .
\end{aligned}
$$

For the special case where $\left(X^{n}, Y^{n}\right)$ is a pair of random vectors with i.i.d components, we recall from [1] that for any $\theta \geq 0$

$$
\lim _{n \rightarrow \infty} \varphi_{n}(\theta)=\varphi(\theta)=\ln \sum_{y}\left[\sum_{x} P(x, y)^{1 /(1+\theta)}\right]^{1+\theta}
$$

This yields the source coding error exponent (with side information $Y^{n}$ ). The well-known source coding error exponent [5, p.37] is obtained by omitting the side information term.

Another special case of interest is when $X^{n}$ represents a codeword from a block code with block length $n$ and rate $R$. Then, $P\left(x^{n}\right)=e^{-n R}$ if $x^{n}$ is a codeword and 0 otherwise. This distribution is called the code's empirical distribution and denoted $Q_{n}$ below. The r.v. $Y^{n}$ represents the channel output when $X^{n}$ is transmitted. We recall from [1] that for $\theta \geq 0$,

$$
\varphi_{n}(\theta)=\theta R-n^{-1} E_{0}\left(\theta, Q_{n}\right)+o(n)
$$

where $E_{0}$ is is Gallager's function [6, p. 138] and $o(n)$ is a quantity that goes to zero as $n$ goes to infinity. Proposition 2 now yields the well-known sphere-packing bound for listdecoding.

In the case of $L=0$, which corresponds to ordinary $\mathrm{ML}$ decoding, Proposition 2 may not apply since 0 may not belong in $\boldsymbol{R}_{\varphi^{\prime}}$. In this case, Gärtner-Ellis theorem yields only a lowerbound.

Proposition 3 Let $\left\{\left(X^{n}, Y^{n}\right)\right\}$ be a sequence of input-output pairs for a noisy channel such that $\left\{\varphi_{n}\right\}$ converges to a limit $\varphi$. Then,

$$
\liminf _{n \rightarrow \infty} n^{-1} \ln P\left[\ln G\left(X^{n} \mid Y^{n}\right)>0\right] \geq-\theta_{0} \varphi_{+}^{\prime}\left(\theta_{0}\right)
$$

where $\theta_{0}=\inf \{\theta: \varphi(\theta)>0\}$ and $\varphi_{+}^{\prime}$ denotes right-derivative.

It can be shown that this bound is equivalent to the familiar sphere-packing lower bound [6, p. 157], except it is formulated in terms of code empirical distributions.

\section{REFERENCES}

[1] E. Arikan, "An inequality on guessing and its application to sequential decoding," IEEE Trans. Inform. Theory, vol. IT-42, no. 1, pp. 99-105, January 1996.

[2] E. Arikan and N. Merhav, "Guessing subject to distortion," IEEE Trans. Inform. Theory, vol. IT-44, no. 3, pp. 1041-1056, May 1998.

[3] E. Arikan and N. Merhav, "Joint source-channel coding and guessing with application to sequential decoding," IEEE Trans. Inform. Theory, vol. IT-44, no. 5, pp. 1756-1769, September 1998.

[4] J. A. Bucklew, Large Deviation Techniques in Decision, Simulation, and Estimation. New York: Wiley, 1990.

[5] I. Csiszár and J. Körner, Information Theory: Coding Theorems for Discrete Memoryless Systems. New York: Academic, 1981.

[6] R. G. Gallager, Information Theory and Reliable Communication. New York: Wiley, 1968. 